Dynamical properties of granular rotors

Non Peer-reviewed author version

CLEUREN, Bart \& EICHHORN, Ralf (2008) Dynamical properties of granular rotors. In: JOURNAL OF STATISTICAL MECHANICS-THEORY AND EXPERIMENT, 2008(10), (ART No P10011).

DOI: $10.1088 / 1742-5468 / 2008 / 10 / P 10011$

Handle: http://hdl.handle.net/1942/9349 


\title{
Dynamical properties of granular rotors
}

\author{
Bart Cleuren $^{1}$ and Ralf Eichhorn ${ }^{2}$ \\ ${ }^{1}$ Hasselt University, B-3590 Diepenbeek, Belgium \\ ${ }^{2}$ Universität Bielefeld, Fakultät für Physik, 33615 Bielefeld, Germany \\ E-mail: bart.cleuren@uhasselt.be, ralf.eichhorn@physik.uni-bielefeld.de
}

\begin{abstract}
The stochastic motion of an arbitrarily shaped rotor, free to rotate around a fixed axis as a result of dissipative collisions with a surrounding thermalized gas, is investigated. A Boltzmann master equation is derived, starting from the elementary gas-rotor collisions. Analytical expressions for the moments of the rotational speed and the rotational temperature are obtained in the form of a series expansion, using the mass ratio of gas particle and rotor as expansion parameter. We discuss the general features of the motion, which is largely determined by the geometry of the rotor. In particular, a stationary non-zero rotation is observed for rotationally asymmetric rotors. Molecular dynamics simulations support the analytical results.
\end{abstract}

Keywords: granular systems, kinetic theory, Brownian motion

PACS numbers: 05.40.-a, 05.70.Ln, 45.70.-n 


\section{Contents}

1 Introduction 2

2 Model description $\quad 3$

3 Analytical results $\quad 6$

3.1 Angular velocity moments . . . . . . . . . . . . . . . . . 6

3.2 Symmetries . . . . . . . . . . . . . . . . . . . 8

3.3 Langevin description . . . . . . . . . . . . . . . . . . . 9

4 Applications $\quad 9$

4.1 Square, rectangle and more symmetric rotors . . . . . . . . . . . . . . . . 10

4.2 Equilateral triangle . . . . . . . . . . . . . . . . . . . . . 11

$\begin{array}{lll}5 & \text { Simulations } & 12\end{array}$

6 Conclusions $\quad 14$

$\begin{array}{ll}\text { Acknowledgments } & 15\end{array}$

$\begin{array}{ll}\text { Appendix. Expansion procedure } & 15\end{array}$

$\begin{array}{ll}\text { References } & 16\end{array}$

\section{Introduction}

Granular gases form an important class of non-equilibrium systems [1]-[3]. Because of the dissipative nature of the particle collisions, they behave quite differently than their elastic counterparts [4]. Most notably, when the gas is left on its own, its temperature will steadily decrease until finally all motion has gone. In order to reach a stationary regime, a constant input of energy is required, for example by shaking the enclosing box. Such a setup in turn may lead to many intricate phenomena, such as granular ratchets [5]-[8], temperature anisotropy [9] or non-Maxwellian velocity distributions [10, 11].

The study of "tracer" systems, consisting of a single granular object interacting dissipatively with a surrounding thermalized bath, has proven to be a convenient tool to reveal properties of granular gases [12]-[17]. For spherical tracer objects in a gas with elastic inter-particle collisions it has been shown that the velocity distribution is Maxwellian $[12,13]$, irrespective of the nature of the collisions between tracer and gas particles (elastic or inelastic). In contrast, non-trivially shaped, anisotropic tracers may show significant deviations from a Maxwellian (angular) velocity distribution [16, 17] under inelastic collisions with the surrounding (elastic) gas.

A connection to Brownian motors [18] is established, whenever these deviations in the velocity distribution are accompanied by a non-vanishing first moment [19, 20]. 
The corresponding directed motion of the tracer particle is generated by rectifying the fluctuations which arise from the random collisions with the surrounding gas. For such a rectification of unbiased random fluctuations to occur, breaking of both spatial and time symmetry is required [18]. Precisely these ingredients are present when an asymmetric tracer object is placed into a granular gas. As a result of the dissipation during collision events, the environment constantly loses energy, part of which is converted into the directed motion of the tracer. Since such motion persists even if working against a (not too large) load force, the asymmetric tracer can operate as a motor [19].

In this work, we investigate the rotational motion of an arbitrarily shaped tracer object (granular rotor) around a fixed axis during incessant dissipative collisions with a surrounding gas. As is to be expected, the shape has a significant influence on the dynamical properties of the object. The (angular) velocity distribution deviates from the Maxwellian form, as is shown indirectly by calculation of the angular velocity moments. A similar conclusion was reached in $[16,17]$ for the case of a granular needle, and it was shown there that the deviations are significant mainly in the limit of small moment of inertia. The results presented here, valid for arbitrary shapes, show that even for large moment of inertia deviations can be observed, most notably through the appearance of a nonzero first moment for rotationally asymmetric rotors. Apart from the theoretical issues of granular matter, and in the context of Brownian motors, rotational motion (being restricted in space) is of relevance to future applications. This is nicely illustrated, e.g., by recent theoretical and simulation work regarding the rotational and dynamical properties of nanosized systems formed by carbon nanotubes [21, 22].

The paper is organized as follows. In section 2 the basic setup is introduced, and the Boltzmann master equation describing the stochastic motion of the rotor is derived. A discussion is given on its validity and the necessary conditions which are assumed. The main results are presented in section 3, clarifying the influence of the geometry on the motion of the rotor. In section 4 the general theory is applied to a number of specific rotor shapes. The theoretical predictions are compared with molecular dynamics simulations in section 5. Finally, the main results are summarized in section 6 , together with a few concluding remarks.

\section{Model description}

The basic setup is shown in figure 1: an object of mass $M$ (henceforth called the rotor) can rotate freely around a fixed axis, as a result of collisions with the surrounding gas particles (mass $m$ ). Our focus here is on a two dimensional situation, with the motion of the rotor and gas particles taking place in the $x-y$ plane orthogonal to the rotational axis. The motion of the rotor is then described by its instantaneous angular velocity $\vec{\omega}=\omega \vec{e}_{z}$. The dynamical properties of the rotor are further specified by its moment of inertia $I$ around the rotational axis, and its geometrical shape. The extension to a general three dimensional rotor is straightforward, although the algebra is considerably more involved. 
The motion of the rotor is induced solely by the interaction with the gas particles. In between collisions, the angular velocity remains constant. The collisions with the rotor are assumed to be instantaneous and inelastic, and described by the (normal) restitution coefficient $0 \leq \alpha<1$ (with $\alpha=1$ corresponding to elastic collisions). The surfaces of both rotor and gas particle are taken to be smooth, implying the absence of tangential forces. The effect of a single collision event follows by applying the appropriate dynamical laws, which in the present situation are (post-collisional quantities are labeled with $\left.\mathrm{a}^{\prime}\right)$ :

- Conservation of angular momentum (no external forces are present):

$$
m \vec{r} \times \vec{v}^{\prime}+I \omega^{\prime}=m \vec{r} \times \vec{v}+I \omega .
$$

- Conservation of tangential velocity of the gas particle:

$$
\vec{v}^{\prime} \cdot \hat{t}=\vec{v} \cdot \hat{t} .
$$

- Inelastic reflection of the normal component of the relative velocity:

$$
\left(\vec{v}^{\prime}-\vec{V}^{\prime}\right) \cdot \hat{n}=-\alpha(\vec{v}-\vec{V}) \cdot \hat{n},
$$

where $\vec{V}=\vec{\omega} \times \vec{r}$ is the velocity of the rotor at the impact position.

The point of impact is specified by its spatial position $\vec{r}=(x, y)$ and the orientation of the surface, determined by the angle $\varphi$ between the $x$-axis and the tangential unit vector $\hat{t}=(\cos \varphi, \sin \varphi)$. The normal to the surface is then $\hat{n}=(\sin \varphi,-\cos \varphi)$. Solving equations (1)-(3) for $\omega^{\prime}$ gives:

$$
\omega^{\prime}=\omega+(1+\alpha) \frac{\varepsilon^{2} g}{R_{I}} \frac{(\vec{V}-\vec{v}) \cdot \hat{n}}{1+\varepsilon^{2} g^{2}},
$$

with $\varepsilon:=\sqrt{m / M}$, and $R_{I}:=\sqrt{I / M}$ the radius of inertia. The geometrical features of the collision, i.e. the dependence on the rotor shape and the position of the rotational axis, come into play through the dimensionless quantity $g$, defined as:

$$
g=g(\vec{r}):=(\vec{r} \cdot \hat{t}) / R_{I}=(x \cos \varphi+y \sin \varphi) / R_{I},
$$

which depends on the point of impact $\vec{r}$ on the rotor surface (for the sake of simplicity, the argument $\vec{r}$ is omitted in the following). As will become clear later, $g$ plays an important role in characterizing the effect of the rotor shape on its dynamical properties. Notice that it does not depend on the details of the surrounding gas, but is solely a property of the rotor.

As a result of the collisions, the angular velocity of the rotor changes stochastically in time. In general, recollisions introduce correlations between these angular velocity changes, which we neglect in the following theoretical analysis, i.e. different collisions are assumed to occur independently. The justification for doing so rests ultimately upon a comparison with molecular simulations or experiments. The dynamics of $\omega$ is then Markovian, and the time evolution of the probability distribution $P(\omega, t)$ is given by a Boltzmann-master equation:

$$
\frac{\partial}{\partial t} P(\omega, t)=\int\left[W\left(\omega \mid \omega^{\prime}\right) P\left(\omega^{\prime}, t\right)-P(\omega, t) W\left(\omega^{\prime} \mid \omega\right)\right] \mathrm{d} \omega^{\prime}
$$


(a)

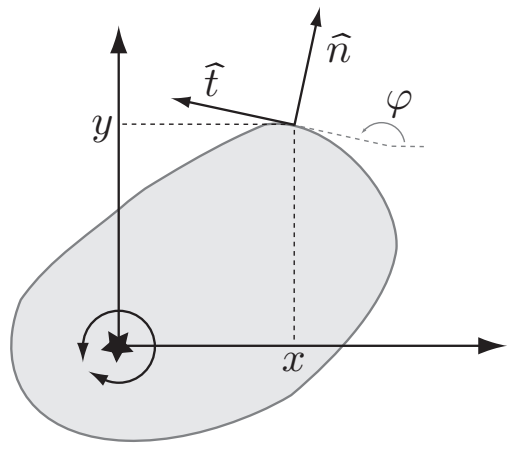

(b)

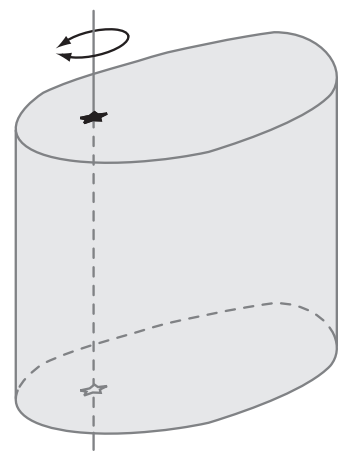

Figure 1. Sketch of the system under consideration: a solid object is free to rotate along a fixed axis, as a result of the collisions with the surrounding gas particles. (a) Top view of the object representing the 2-dimensional reduction used for the theoretical analysis. The coordinate system is placed such that the $z$ axis coincides with the rotation axis, i.e. the center of rotation in the 2-dimensional $x-y$ plane (indicated by the star) coincides with the origin of the coordinate system. A surface point of the 2 -dimensional (reduced) object is given by $\vec{r}=(x, y)$; its normal unit vector $\hat{n}$ and its tangential unit vector $\hat{t}$ as well as the angle $\varphi$ with the positive $x$ axis are also shown. (b) Total view of the object, which is homogeneous along the axis of rotation, and thus allows for a reduction to 2 dimensions.

with $W\left(\omega^{\prime} \mid \omega\right)$ being the probability per unit time for the angular velocity to change from $\omega$ to $\omega^{\prime}$. It is given by:

$$
\begin{aligned}
W\left(\omega^{\prime} \mid \omega\right)= & \int \mathrm{d} S \int_{-\infty}^{+\infty} \mathrm{d} v_{x} \int_{-\infty}^{+\infty} \mathrm{d} v_{y} \rho \phi\left(v_{x}, v_{y}\right) \Theta[(\vec{V}-\vec{v}) \cdot \hat{n}] \\
& \times|(\vec{V}-\vec{v}) \cdot \hat{n}| \delta\left[\omega^{\prime}-\omega-(1+\alpha) \frac{\varepsilon^{2} g}{R_{I}} \frac{(\vec{V}-\vec{v}) \cdot \hat{n}}{1+\varepsilon^{2} g^{2}}\right]
\end{aligned}
$$

The integral over the surface $S$ of the rotor takes into account all the possible collision points at the surface of the rotor. The Dirac delta function picks out the velocities which give the corresponding change $\omega^{\prime}-\omega$ of the rotational speed. $\phi\left(v_{x}, v_{y}\right)$ represents the velocity distribution of the surrounding gas, taken to be by the Maxwell-Boltzmann distribution at temperature $T$. Integrating out the velocity components of the incoming particle leads to:

$$
\begin{aligned}
W\left(\omega^{\prime} \mid \omega\right)= & \frac{\rho}{(1+\alpha)^{3}} \sqrt{\frac{I^{2}}{2 \pi m k T}} \int \mathrm{d} S\left|\omega^{\prime}-\omega\right| \frac{\left(1+\varepsilon^{2} g^{2}\right)^{2}}{\varepsilon^{2} g^{2}} \\
& \times \Theta\left[\frac{\omega^{\prime}-\omega}{g}\right] \exp \left[-\frac{I}{2 k T}\left(\omega \varepsilon g+\frac{\left(\omega^{\prime}-\omega\right)\left(1+\varepsilon^{2} g^{2}\right)}{(1+\alpha) \varepsilon g}\right)^{2}\right]
\end{aligned}
$$

This expression, together with the master equation (6), yields an exact microscopic description of the rotational motion of the rotor under the given assumptions, and constitutes the starting point for its analysis.

The basic premises of our theory - uncorrelated collisions with Maxwell-Boltzmann distributed gas particles - are justified if recollisions are rare. Then, correlations 
between different collisions can safely be neglected, and the distribution of incoming particles colliding with the rotor does not deviate noticeably from the MaxwellBoltzmann distribution. As confirmed by molecular dynamics simulations (section 5) these conditions are very well fulfilled for convex shaped rotors in a diluted thermalized gas.

\section{Analytical results}

\subsection{Angular velocity moments}

The central quantities of interest are the moments of the angular velocity in the stationary regime $t \rightarrow \infty$, given by

$$
\left\langle\omega^{n}\right\rangle:=\lim _{t \rightarrow \infty} \int \mathrm{d} \omega \omega^{n} P(\omega, t) .
$$

However, an explicit exact solution of (6) is not possible, not even in the steady state regime $\partial_{t} P(\omega, t)=0$ approached for large times, and we must resort to a perturbative method. In most cases of interest, the mass of the rotor is much larger than the gas particle mass, $M \gg m$. Then, a systematic expansion of the Master equation in the small parameter $\varepsilon=\sqrt{m / M}$ is feasible, similar in spirit as Van Kampen's system-size expansion [23]. Starting from the Boltzmann master equation (6) one can derive the (infinite) set of coupled evolution equations for the moments. These equations can be decoupled by a series expansion in $\varepsilon$. In the steady state $\left(\partial_{t}\left\langle\omega^{n}\right\rangle=0\right)$ the solution reduces to a (tedious) algebraic problem. The result is an analytical expression for all moments $\left\langle\omega^{n}\right\rangle(n=1,2, \ldots)$ in the form of a series expansion in terms of $\varepsilon$. An outline of the procedure is given in the Appendix, see also the discussion in reference [24].

Of particular physical interest for analyzing the motion of the rotor are the first two moments, namely the average rotational speed $\langle\omega\rangle$ and its "fluctuations" $\left\langle\omega^{2}\right\rangle$. Their explicit expressions, showing first two terms in the series expansion (order $\varepsilon^{3}$ for $\langle\omega\rangle$ and $\varepsilon^{4}$ for $\left.\left\langle\omega^{2}\right\rangle\right)$, are given by:

$$
\begin{aligned}
\langle\omega\rangle= & \left(\frac{m}{M}\right)^{1 / 2} \sqrt{\frac{\pi k T}{2 I}} \frac{\alpha-1}{4} \frac{\left\langle g^{3}\right\rangle}{\left\langle g^{2}\right\rangle} \\
& +\left(\frac{m}{M}\right)^{3 / 2} \sqrt{\frac{\pi k T}{2 I}} \frac{\alpha-1}{32}\left[2(\alpha+5) \frac{\left\langle g^{3}\right\rangle\left\langle g^{4}\right\rangle}{\left\langle g^{2}\right\rangle^{2}}-8 \frac{\left\langle g^{5}\right\rangle}{\left\langle g^{2}\right\rangle}-\pi \frac{\left\langle g^{3}\right\rangle^{3}}{\left\langle g^{2}\right\rangle^{3}}\right]+\ldots \\
\left\langle\omega^{2}\right\rangle= & \frac{k T}{I} \frac{\alpha+1}{2}+\frac{m}{M} \frac{k T}{I} \frac{\alpha-1}{16}\left[4(\alpha+1) \frac{\left\langle g^{4}\right\rangle}{\left\langle g^{2}\right\rangle}-\pi \frac{\left\langle g^{3}\right\rangle^{2}}{\left\langle g^{2}\right\rangle^{2}}\right]+\ldots
\end{aligned}
$$

The powers of $g$ in brackets are defined as average over the (one-dimensional) surface of the rotor:

$$
\left\langle g^{n}\right\rangle:=\int \frac{\mathrm{d} S}{S} g^{n}
$$

and contain the detailed information about its geometry. 
When additionally taking into consideration the third and fourth moment, $\left\langle\omega^{3}\right\rangle$ and $\left\langle\omega^{4}\right\rangle$, we can quantitatively compare the distribution of the angular rotor velocity with a Gaussian (Maxwellian) form by determining the skewness

$$
\zeta:=\frac{\left\langle(\omega-\langle\omega\rangle)^{3}\right\rangle}{\left\langle(\omega-\langle\omega\rangle)^{2}\right\rangle^{3 / 2}}
$$

and kurtosis excess

$$
\kappa:=\frac{\left\langle(\omega-\langle\omega\rangle)^{4}\right\rangle}{\left\langle(\omega-\langle\omega\rangle)^{2}\right\rangle^{2}}-3
$$

which both vanish in case of a Gaussian distribution. In lowest non-trivial order (being $\varepsilon^{3}$ for $\zeta$ and $\varepsilon^{4}$ for $\left.\kappa\right)$ we obtain:

$$
\begin{aligned}
& \zeta=\left(\frac{m}{M}\right)^{3 / 2} \sqrt{\pi(\alpha+1)} \frac{\alpha-1}{4}\left[\frac{\left\langle g^{5}\right\rangle}{\left\langle g^{2}\right\rangle}-\frac{7\left\langle g^{3}\right\rangle\left\langle g^{4}\right\rangle}{6\left\langle g^{2}\right\rangle^{2}}+\frac{\pi\left\langle g^{3}\right\rangle^{3}}{8\left\langle g^{2}\right\rangle^{3}}\right]+\ldots \\
& \kappa=\left(\frac{m}{M}\right)^{2} \frac{\alpha^{2}-1}{4}\left[\frac{\left\langle g^{6}\right\rangle}{\left\langle g^{2}\right\rangle}-\frac{4\left\langle g^{4}\right\rangle^{2}+3 \pi\left\langle g^{3}\right\rangle\left\langle g^{5}\right\rangle}{4\left\langle g^{2}\right\rangle^{2}}+\frac{\pi\left\langle g^{3}\right\rangle^{2}\left\langle g^{4}\right\rangle}{\left\langle g^{2}\right\rangle^{3}}-\frac{3 \pi^{2}\left\langle g^{3}\right\rangle^{4}}{32\left\langle g^{2}\right\rangle^{4}}\right]+\ldots
\end{aligned}
$$

The expressions (10), (11) and (15), (16) constitute the main analytical results of the present work. We can immediately infer (within the presented order in $\varepsilon$ ) that the tracer object is at equilibrium with the surrounding gas, independently of its shape, for elastic collisions $(\alpha=1)$ with the gas particles, since then the angular velocity distribution is Maxwellian with the gas temperature $T$. Furthermore, these expressions also predict a systematic rotation of the tracer object due to a non-vanishing first moment $\langle\omega\rangle$, accompanied by an asymmetric distribution of the angular velocity, provided the following two conditions are fulfilled: (i) a restitution coefficient smaller than unity, $\alpha<1$, and (ii) a nonzero $\left\langle g^{3}\right\rangle$ (in lowest order).

The first condition implies that the system is necessarily out of equilibrium. This can be seen by defining the temperature $k T_{\mathrm{r}}=\left\langle\omega^{2}\right\rangle I$ that the rotor adopts from the interaction with the gas. From (11) we read off:

$$
T_{\mathrm{r}}=T\left(\frac{\alpha+1}{2}+\frac{m}{M} \frac{\alpha-1}{16}\left[4(\alpha+1) \frac{\left\langle g^{4}\right\rangle}{\left\langle g^{2}\right\rangle}-\pi \frac{\left\langle g^{3}\right\rangle^{2}}{\left\langle g^{2}\right\rangle^{2}}\right]+\ldots\right) .
$$

In lowest order this expression becomes independent on the rotor's shape and reduces to the result $T_{\mathrm{r}}=T(\alpha+1) / 2$, which turns out to be quite general and not limited to $M \gg m$ [12]. For dissipative collisions with $\alpha<1$ the rotor temperature $T_{\mathrm{r}}$ is smaller than $T$. Because of this temperature difference, an energy flow is established from the gas into the object. Replacing $\alpha$ in (10) in terms of $T$ and $T_{r}$, reveals that $\langle\omega\rangle \propto T-T_{\mathrm{r}}$ in lowest order.

The second condition, $\left\langle g^{3}\right\rangle \neq 0$ is of purely geometrical origin, and occurs when the object breaks the rotational symmetry. We will comment on this statement further in the next section.

As is well known, these two conditions - breaking of detailed balance and spatial symmetry - are necessary ingredients for the appearance of a Brownian motor [18], implying that the here analyzed granular rotor may operate as a granular motor. A specific example is presented in section 4. Analogous results were obtained in $[19,20]$ for the case of translational motion. 


\subsection{Symmetries}

As already mentioned above, in the results (10), (11) and (15), (16), the information about the shape of the rotor and the location of the rotation center is exclusively $\ddagger$ contained in the surface averages $\left\langle g^{n}\right\rangle$ as defined in (12). We thus concentrate on their properties first and show that they obey the following three symmetries:

(i) $\langle g\rangle=0$ independent of the rotor's shape.

(ii) $\left\langle g^{n}\right\rangle$ is invariant under rotation around the rotor's center of rotation.

(iii) $\left\langle g^{n}\right\rangle \rightarrow(-1)^{n}\left\langle g^{n}\right\rangle$ for $x \rightarrow-x$ (or $y \rightarrow-y$ ).

The proofs are straightforward:

(i) In the case $n=1$ we can choose $\mathrm{d} \vec{r}:=\hat{t} \mathrm{~d} S$ to find $\langle g\rangle \propto \oint \vec{r} \cdot \mathrm{d} \vec{r}$. For any physical object, the path along which this line integral is calculated will be closed. We can then apply Stokes' theorem which leads to the surface integral $\iint \nabla \times \vec{r} \cdot \mathrm{d} \vec{O}$, and hence $\langle g\rangle=0$ independent of the object's shape and the location of the center of rotation. Actually, this identity has already been incorporated in the results (10), (11) and (15), (16).

(ii) The rotation invariance immediately follows from the fact that $g$ is defined by the scalar product (5) in a coordinate system where the origin coincides with the center of rotation, and that $R_{I}$ depends on the rotation center only via the moment of inertia $I$.

(iii) When reflecting the rotor at the $y$-axis, the location of a surface element at $\vec{r}=(x, y)$ is moved to $(-x, y)$. Correspondingly, the angle $\varphi$ defining the normal (pointing outwards) and tangential vectors to this surface element transforms into $2 \pi-\varphi$. From (5), we thus find $g \rightarrow-g$ under $x \rightarrow-x$ (recalling that $I$, and thus $R_{I}$, is invariant under reflection), and the above statement $\left\langle g^{n}\right\rangle \rightarrow(-1)^{n}\left\langle g^{n}\right\rangle$ readily follows. Analogous arguments apply for the transformation $y \rightarrow-y$ when the rotor is mirrored at the $x$ axis.

The most important result is (iii). Together with (10) and (11) it follows that the rotor, if reflected at one of the coordinate axes, switches rotation direction $\langle\omega\rangle$, whereas the amount of "fluctuations" in the angular velocity $\left\langle\omega^{2}\right\rangle$ remain unchanged. These findings reproduce the fundamental mirror symmetry $\left\langle\omega^{n}\right\rangle \rightarrow(-1)^{n}\left\langle\omega^{n}\right\rangle$ for the moments of the angular velocity, which is required to be valid for physical reasons due to the symmetry of our setup. Put differently, any order of the series expansion of $\left\langle\omega^{n}\right\rangle$ in $\varepsilon$ must have a functional structure in terms of $\left\langle g^{n}\right\rangle$ that preserves this mirror symmetry by the property (iii); the results (10) and (11) constitute examples of this statement for $\langle\omega\rangle$ and $\left\langle\omega^{2}\right\rangle$ to order $\varepsilon^{3}$. Analogous arguments apply to the functional structure of the skewness $\zeta$ and the kurtosis excess $\kappa$, see equations (15) and (16).

As an immediate and central further consequence we find:

$$
\begin{array}{ll}
\langle\omega\rangle=0 & \text { if the rotation center is located on a } \\
& \text { mirror symmetry axis of the rotor. }
\end{array}
$$

$\ddagger$ Actually, the moment of inertia $I$ does depend on the position of the rotation center. However, $I$ trivially is invariant under rotation and reflection, and thus this dependence is of no relevance for what we are going to prove. 
The reason is that the rotor and the center of rotation are mapped onto itself upon reflection at a mirror axis, so that $\langle\omega\rangle$ remains unchanged, in addition to $\langle\omega\rangle \rightarrow-\langle\omega\rangle$ from the reflection symmetry. More intuitively, the clockwise and counter-clockwise rotational movements around a center that is fixed on a mirror axis of the object are performed in identical physical manners, and thus exactly cancel. In this sense, one has to break "rotational symmetry" of the setup by breaking the mirror symmetry of the rotor or by moving the rotation center away from the mirror axis, in order to get a non-vanishing average angular motion. On the level of the geometric quantities $\left\langle g^{n}\right\rangle$, (18) is tantamount to $\left\langle g^{n}\right\rangle=0$ for $n$ odd (see the symmetry (iii) above), so that the spatial asymmetry with respect to rotation manifests itself in $\left\langle g^{n}\right\rangle \neq 0$ for $n$ odd.

\subsection{Langevin description}

By means of a Kramers-Moyal expansion one can derive a Fokker-Planck equation from the master equation (6) (see the Appendix), and by identifying drift and diffusion terms one ends up with the following Langevin equation in lowest order of $\varepsilon$ :

$$
\dot{\omega}(t)=-\frac{\gamma}{I} \omega(t)+\frac{F}{I}+\eta(t)
$$

with the friction coefficient $\gamma$ and the external torque $F$ given by:

$$
\begin{aligned}
& \gamma=(\alpha+1) \rho \sqrt{\frac{2 k T m}{\pi}} S R_{I}^{2}\left\langle g^{2}\right\rangle \\
& F=\frac{\left(\alpha^{2}-1\right)}{4} \rho \frac{k T m}{I} S R_{I}^{3}\left\langle g^{3}\right\rangle
\end{aligned}
$$

and $\eta$ being the white noise term characterized by:

$$
\langle\eta(t)\rangle=0 \quad \text { and } \quad\left\langle\eta(t) \eta\left(t^{\prime}\right)\right\rangle=\frac{2 \gamma k T}{I^{2}} \frac{\alpha+1}{2} \delta\left(t-t^{\prime}\right)
$$

This Langevin description gives a intuitive picture of the stochastic behaviour of the granular rotor. The rotor is, on the one hand, driven by a constant forcing, which is proportional to the gas temperature and emerges from the inelastic collisions with the gas particles $(\alpha<1)$. On the other hand, these collisions also induce friction and Brownian random forces being present independent of the collisions' nature. Note that the friction coefficient scales with the square root of the gas temperature $T$, as well known from the kinetic theory of gases [25]. Moreover, both the friction (20) and the forcing (21) are proportional to the gas density $\rho$, so that the average angular velocity $\langle\omega\rangle=F / \gamma$ becomes density-independent, in agreement with (10). In lowest order of $\varepsilon$, the results (10) and (11) for $\langle\omega\rangle$ and $\left\langle\omega^{2}\right\rangle$ are easily reproduced from the Langevin equation (19).

\section{Applications}

Having established the general framework, we now continue with applying it to specific rotor shapes. We focus on (convex) rotors that are built of straight linear segments 
(a)

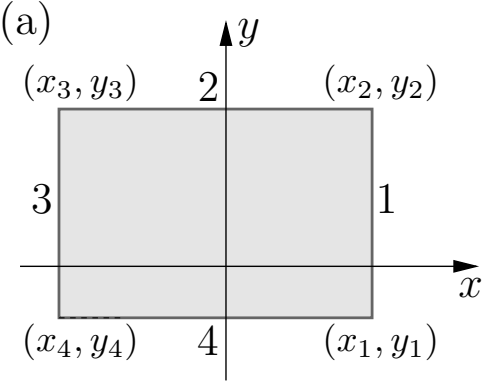

(b)

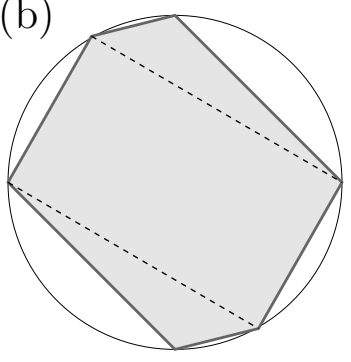

Figure 2. (a) Rectangle with its sided parallel to the coordinate axes; the origin of the coordinate system represents the center of rotation. (b) Highly symmetric rotor with all corners being located ion a circle and with pair of opposite sides of equal length that can be imagined to belong to rectangles, as indicated by the dashed lines for one pair of opposite sides.

("sides"), see figures 2 and 3 for illustration. Before turning to some explicit examples, we discuss a large class of highly symmetric piecewise linear rotors that do not rotate at all.

\subsection{Square, rectangle and more symmetric rotors}

For rotor shapes consisting of straight segments, the surface integral (12) can be rewritten as a sum over those sides:

$$
\left\langle g^{n}\right\rangle=\sum_{\text {side } i} \int \frac{\mathrm{d} S_{i}}{S} g_{i}^{n} .
$$

Any term in this sum is invariant under rotation around the center of rotation, as follows from the same argument used for demonstrating the symmetry (ii) above. This invariance expresses the physical consequence of the isotropicity of the granular gas: the contribution of the impacts of gas particles on any side of the rotor to its average rotational speed $\langle\omega\rangle$ (and to the higher moments of $\omega$ as well) is independent of the actual angular position of the rotor.

When calculating $\left\langle g^{n}\right\rangle$ for the rectangular rotor of figure $2 \mathrm{a}$, it is thus most convenient to rotate the rectangle into a position where its sides are parallel to the coordinate axes, because then the functions $g_{i}$ and the surface elements $\mathrm{d} S_{i}$ in (23) have a very simple form. For instance, for the vertical side 1 in figure 2a, one readily finds $\left\langle g_{1}^{n}\right\rangle=\int_{y_{1}}^{y_{2}} \mathrm{~d} y / S y^{n}$, and for the opposite side $3\left\langle g_{3}^{n}\right\rangle=\int_{y_{4}}^{y_{3}} \mathrm{~d} y / S(-y)^{n}$. In view of $y_{1}=y_{4}$ and $y_{2}=y_{3}$ (see figure 2a), the sum of these surface averages gives $\left\langle g_{1}^{n}\right\rangle+\left\langle g_{3}^{n}\right\rangle=0$ for $n$ odd. This result is completely independent of the position of the center of rotation, because the integrals are independent of $x$ anyway, and a translation of the origin along the $y$ direction would only shift the integration limits $y_{1}, y_{2}, y_{3}$ and $y_{4}$ by the same distance without changing the identities $y_{1}=y_{4}$ and $y_{2}=y_{3}$. (Note, however, that the sum $\left\langle g_{1}^{n}\right\rangle+\left\langle g_{3}^{n}\right\rangle=2\left\langle g_{1}^{n}\right\rangle$ for $n$ even does depend on the rotation center.) The same result is readily found for the two other opposite rectangle sides 2 and 4 (see figure 2a), either 


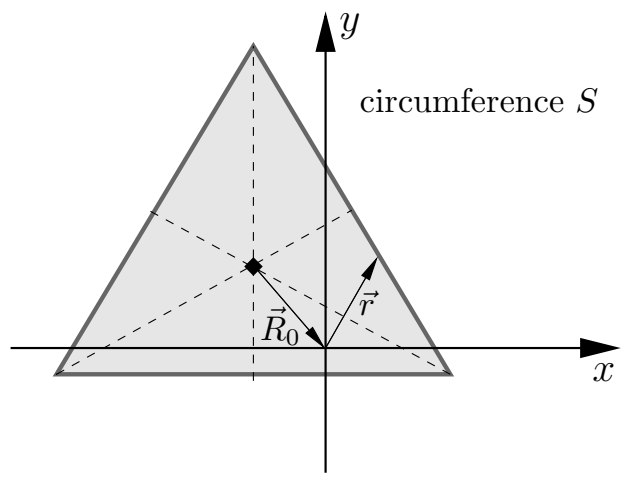

Figure 3. Equilateral triangle with equal sides, each of length $S / 3$. The center of rotation coincides with the origin of the $(x, y)$ coordinate system and is positioned at $\vec{R}_{0}=\left(X_{0}, Y_{0}\right)$ with respect to the center of mass (the diamond in the middle of the triangle). The dashed lines represent the mirror symmetry lines of the triangle.

by direct calculation or else by exploiting the rotation invariance of $\left\langle g_{i}^{n}\right\rangle$. If applied to the result for the rectangle sides 1 and 3 the latter tells us that

$$
\begin{aligned}
\left\langle g_{i}^{n}\right\rangle+\left\langle g_{j}^{n}\right\rangle=0 & (n \text { odd }) \\
& \text { for any pair } i, j \text { of opposite sides } \\
& \text { that belong to a rectangle. }
\end{aligned}
$$

We immediately infer that $\left\langle g^{n}\right\rangle=0$ ( $n$ odd) for squares and rectangles, and thus $\langle\omega\rangle=0$ [see equation (10) and the discussion in section 3.2 following the proofs of the symmetries (i)-(iii)], irrespective of the location of the rotation center. However, (24) implies the absence of systematic rotation for a much larger class of rotors: Any object that consists of an even number of sides where opposite sides have equal length and with corners that are all located on a circle, does not rotate, i.e. $\langle\omega\rangle=0$. The reason is that such an object can be imagined to be composed of rectangles, see figure $2 \mathrm{~b}$ for an illustration. Of course, the rectangle itself is a special case with just four sides.

\subsection{Equilateral triangle}

As a concrete rotor we consider in the following the simplest non-trivial case of an equilateral triangle, see figure 3. Since we are interested in the dependence of the angular velocity on the position of the rotation center, we specify its location by $\vec{R}_{0}=\left(X_{0}, Y_{0}\right)$ with respect to the center of mass.

Performing the surface averages (12) for $n$ up to 5 , we find

$$
\begin{aligned}
& \left\langle g^{2}\right\rangle=\frac{1}{108 R_{I}^{2}}\left[S^{2}+54\left(X_{0}^{2}+Y_{0}^{2}\right)\right], \\
& \left\langle g^{3}\right\rangle=-\frac{1}{4 R_{I}^{3}} X_{0}\left(X_{0}^{2}-3 Y_{0}^{2}\right), \\
& \left\langle g^{4}\right\rangle=\frac{1}{6480 R_{I}^{4}}\left[S^{4}+180 S^{2}\left(X_{0}^{2}+Y_{0}^{2}\right)+2430\left(X_{0}^{2}+Y_{0}^{2}\right)^{2}\right],
\end{aligned}
$$




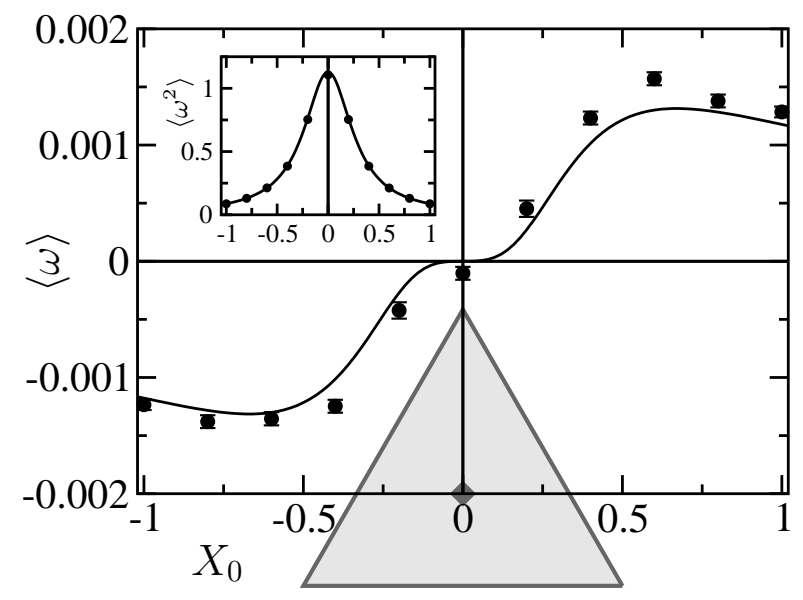

Figure 4. Average rotational speed of the equilateral triangle from figure 3 for $k T=1$, $m=1, \rho=0.0025, M=10, \alpha=0.9, S=3, Y_{0}=0$ and for varying $X_{0}$. Inset: Second moment of $\omega$ for the same parameter values. The solid lines show the analytical results from (10) and (11) (inset), with (25a)-(26b), whereas the dots with error bars are the results of molecular dynamics simulations (in the inset, the error bars are smaller than the symbol sizes). The grey triangle is positioned in a way that the $X_{0}$ axis represents the locations of the rotation axis with respect to the triangle and its center of mass, which is marked by a grey diamond.

$$
\left\langle g^{5}\right\rangle=-\frac{5}{432 R_{I}^{5}} X_{0}\left(X_{0}^{2}-3 Y_{0}^{2}\right)\left[2 S^{2}+27\left(X_{0}^{2}+Y_{0}^{2}\right)\right] .
$$

Moreover, by Steiner's theorem we get

$$
\begin{aligned}
& I=M\left(S^{2} / 108+X_{0}^{2}+Y_{0}^{2}\right) \\
& R_{I}=\sqrt{\left(S^{2} / 108+X_{0}^{2}+Y_{0}^{2}\right)} .
\end{aligned}
$$

With these expressions we can calculate the angular velocity $\langle\omega\rangle$ and and its "fluctuations" $\left\langle\omega^{2}\right\rangle$ up to order $\varepsilon^{3}$, from (10) and (11). The results are shown in figure 4 for $Y_{0}=0$ and varying $X_{0}$. Note that the odd averages $\left\langle g^{3}\right\rangle$ and $\left\langle g^{5}\right\rangle$ vanish for $X_{0} \equiv 0$ and $Y_{0}= \pm X_{0} / \sqrt{3}$. These lines constitute the mirror symmetry lines of the triangle, and, as expected, the average rotational speed is indeed zero at these lines according to (10).

Also included in figure 4 are the results of molecular dynamics simulations for the rotating triangle (figure 3), showing remarkably good agreement with the theory. The details behind the simulations are explained in the following section.

\section{Simulations}

The motion of the rotor due to the collisions with surrounding gas particles is simulated by an event-driven molecular dynamics code. The gas is treated as an ideal gas of pointlike particles, i.e. no collisions between the gas particles occur. The collisions of gas particles with the object are detected numerically by solving the exact transcendental 
equations for the point of impact on the rotor surface, which result from equating the rotational motion of the linear rotor sides with the translational gas particle movement. At each collision, the rotational speed of the rotor is changed according to the collision rule (4), and the velocity of the gas particle according to the collision rule obtained from solving equations (1)-(3) for $\vec{v}^{\prime}$.

In order to mimic an infinitely large reservoir of gas particles with given density and temperature, the rotor is placed into a rectangular box which is filled with gas particles according to the prescribed density, and whose boundaries have the following properties: A gas particle that reaches a box wall is taken out of the gas (it exits the box and "disappears" into a surrounding infinite reservoir); new gas particles are created at the box walls according to a Poissonian process (as if they were entering the box from a surrounding gas reservoir) with a rate parameter determined by the gas properties (in particular density and temperature) and the box size. It is taken care, of course, that the box is large enough (typically of size $20 \times 20$ ) to completely incorporate the rotating object.

The presented simulation results (figure 4 and 5) are obtained by simulating the collisions of about 20000 different gas particles with the object, which initially is at rest. For the parameter values given in figure 4, e.g., this corresponds to a simulation time of approximately $7 \times 10^{6}$. Depending on the amount of fluctuations (cf. the results for $\left\langle\omega^{2}\right\rangle$ in figure 4 and 5) averages over 20000 to 1000000 runs are performed.

The actual number of detected collisions for different runs vary significantly due to the regular occurrence of recollisions of gas particles with the rotating object. Such recollisions are obviously correlated and, moreover, introduce deviations of the gas particle velocity distribution from the Maxwellian equilibrium form, in contrast to the assumptions of our theory (see section 2). However, the effects on the average rotational speed of the rotor turn out to be very small for not too large gas densities. This is nicely demonstrated by the excellent agreement between theory and simulation results (for $\rho=0.0025$ ) displayed in figure 4 (see also figure 5a). We conclude that our assumptions are very well justified.

To further test the range of validity of our theory we compare in figure 5 the theoretically predicted rotational speed with simulation results for varying gas density and rotor mass. As already mentioned in section 3.3, the linear dependence of the effective driving force (21) and friction (20) on the gas density $\rho$ cancels out to result in a density independent average rotational speed $\langle\omega\rangle$, cf. (10). Figure 5a shows that this theoretical prediction breaks down at large densities, where the effects of an increased number of recollisions may lead to different (non-linear) $\rho$-dependencies of the driving force and friction coefficient, and imply a larger disagreement with the theoretical prediction. Furthermore, due to the series expansion in $\varepsilon=\sqrt{m / M}$ the deviations between theory and simulations are expected to become more notable for smaller mass ratios $m / M$, see figure $5 \mathrm{~b}$. For $m / M \lesssim 2$ the third order result (10) even gives a qualitatively wrong behaviour as it predicts a decreasing rotational velocity for smaller mass ratios. It can be improved, however, by taking into account higher order terms. 

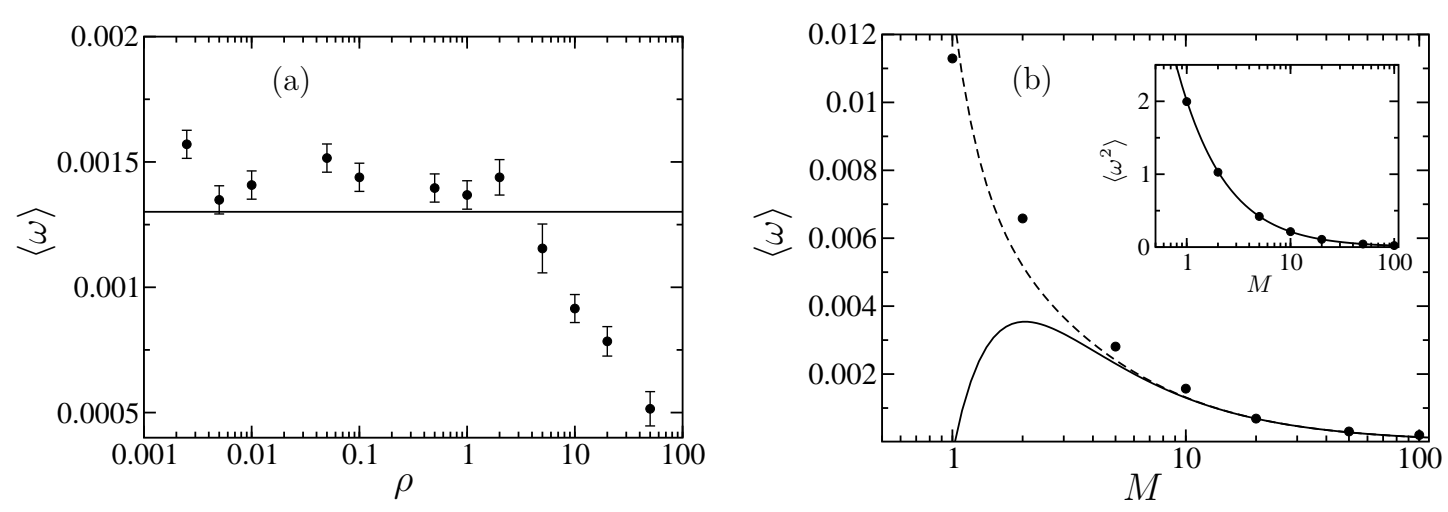

Figure 5. Average rotational speed of the equilateral triangle from figure 3 for $k T=1$, $m=1, \alpha=0.9, S=3, Y_{0}=0, X_{0}=0.6$ (i.e. at approximately maximal average rotational speed, see figure 4). (a) Dependence on the gas density $\rho$ for $M=10$ from the theoretical result (10) with (25a)-(26b) (solid line) and from molecular dynamics simulations (dots with error bars). (b) Dependence on the rotor mass $M$ for $\rho=0.0025$ (inset: $\left\langle\omega^{2}\right\rangle$ as a function of $M$ ) from the theoretical result (10) with (25a)-(26b) (solid line) and from molecular dynamics simulations (dots, the error bars are smaller than the symbol size). The dashed line shows the theoretical result corresponding to (10) with the next order in $\varepsilon=\sqrt{m / M}$ taken into account, i.e. for fifth order in $\varepsilon$.

\section{Conclusions}

We have studied the rotational motion of a heavy granular tracer exposed to inelastic collisions with particles from a surrounding thermalized gas (granular rotor). Explicit results are obtained for the first two moments of the angular velocity, in (10) and (11) and the rotational temperature of the object (equation (17)) in the form of a series expansion, valid for arbitrary rotor shapes. Deviations from the Gaussian form of the angular velocity distribution are quantified by calculating its skewness and kurtosis, see equations (15) and (16). It turns out that such deviations are small in that the results are non-trivial only in higher orders of the series expansion.

For rotationally asymmetric granular rotors a non-vanishing first moment of the angular velocity distribution, representing a non-zero average rotational speed of the tracer, is found if the collisions with the gas particles are inelastic. The result (10) for the average rotational speed clearly indicates the requirements needed to observe a steady rotation: dissipation, meaning that the combined system (gas with rotor) is out of equilibrium, and a spatial asymmetry. As mentioned before, these two ingredients are necessary for the appearance of a systematic motion in Brownian motors [18]. As is discussed previously in the literature [24, 26, 27, 28], non-equilibrium conditions may also be established by putting different parts of the rotor in contact with distinct gases at different temperatures.

We point out that our analytical approach allows for a direct calculation of the velocity moments without any a priori knowledge about the velocity distribution. The obtained results are exact under the assumption that the successive collisions between 
gas particles and tracer object occur independently. In particular, the influence of the rotating object on the gas, changing the gas properties near the surface of the rotor, is neglected. As verified by molecular dynamics simulations, this assumption is very well justified and we expect our theory to yield the dominating contribution to the dynamical behavior of the rotor under quite general conditions, see section 5 .

\section{Acknowledgments}

This work was partially supported by the StochDyn program of the ESF. B.C. acknowledges support from the Fonds voor Wetenschappelijk Onderzoek Vlaanderen. R.E. acknowledges support by the Deutsche Forschungsgemeinschaft (SFB 613).

\section{Appendix. Expansion procedure}

A systematic expansion of the master equation is based on the fact that the change in velocity $\omega^{\prime}-\omega$, as given by (4), is of order $\varepsilon^{2}$. As a result, the transition rate $W\left(\omega^{\prime} \mid \omega\right)$ vanishes rapidly for increasing $\omega^{\prime}-\omega$, justifying our choice of $\varepsilon$ as the expansion parameter. We note, however, that $M$ also appears implicitly in the velocity $\omega$, as anticipated from the equipartition principle $I\left\langle\omega^{2}\right\rangle \propto k T$. Therefore, we switch to the dimensionless quantity $\Omega$, defined as:

$$
\Omega:=\sqrt{\frac{I}{k T}} \omega
$$

A Kramers-Moyal expansion of the master equation (6), in terms of this new variable, gives the following equivalent form:

$$
\partial_{t} P(\Omega, t)=\sum_{n=1}^{\infty} \frac{(-1)^{n}}{n !}\left(\frac{\mathrm{d}}{\mathrm{d} \Omega}\right)^{n}\left[A_{n}(\Omega) P(\Omega ; t)\right],
$$

with $A_{n}(\Omega)$ being the so-called jump moment, defined as:

$$
A_{n}(\Omega)=\sqrt{\frac{k T}{I}} \int \mathrm{d} \Omega^{\prime}\left(\Omega^{\prime}-\Omega\right)^{n} W\left(\sqrt{k T / I} \Omega^{\prime} \mid \sqrt{k T / I} \Omega\right) .
$$

Their explicit expression reads:

$$
\begin{aligned}
A_{n}(\Omega)=2^{\frac{n}{2}} & \rho \sqrt{\frac{k T}{2 \pi m}} \int \mathrm{d} S\left(\frac{\varepsilon g(1+\alpha)}{1+\varepsilon^{2} g^{2}}\right)^{n} e^{-\frac{(\Omega \varepsilon g)^{2}}{2}} \\
\times & {\left[\Gamma(1+n / 2) \Phi\left(1+n / 2,1 / 2 ; \Omega^{2} \varepsilon^{2} g^{2} / 2\right)\right.} \\
& \left.+\sqrt{2} \Omega \varepsilon g \Gamma((3+n) / 2) \Phi\left((3+n) / 2,3 / 2 ; \Omega^{2} \varepsilon^{2} g^{2} / 2\right)\right] .
\end{aligned}
$$

The function $\Phi(a, b, z)$ is Kummer's confluent hypergeometric function.

Of more interest to us is the following set of coupled equations, describing the time evolution of the moments $\left\langle\Omega^{n}\right\rangle:=\int \mathrm{d} \Omega \Omega^{n} P(\Omega, t)$ :

$$
\partial_{t}\langle\Omega\rangle=\left\langle A_{1}(\Omega)\right\rangle
$$




$$
\begin{aligned}
& \partial_{t}\left\langle\Omega^{2}\right\rangle=2\left\langle\Omega A_{1}(\Omega)\right\rangle+\left\langle A_{2}(\Omega)\right\rangle \\
& \partial_{t}\left\langle\Omega^{3}\right\rangle=3\left\langle\Omega^{2} A_{1}(\Omega)\right\rangle+3\left\langle\Omega A_{2}(\Omega)\right\rangle+\left\langle A_{3}(\Omega)\right\rangle \\
& \ldots
\end{aligned}
$$

These equations follow directly from (A.2) by multiplying it with $\Omega^{n}$ and subsequent integration over $\Omega$. This new set of equations are fully coupled and equally difficult to solve as the original Master equation. However, the equations decouple in the limit $\varepsilon=\sqrt{m / M} \rightarrow 0$. Indeed, using the following series expansions:

$$
\Phi\left(\alpha, \gamma ; z^{2}\right)=1+\frac{\alpha}{\gamma} z^{2}+\frac{\alpha(\alpha+1)}{\gamma(\gamma+1)} z^{4}+\ldots
$$

one finds:

$$
A_{1}(\Omega) \approx-(1+\alpha) \rho \sqrt{\frac{k T}{m}}\left\{\sqrt{\frac{2}{\pi}} \Omega\left\langle g^{2}\right\rangle \varepsilon^{2}+\frac{\left(1-\Omega^{2}\right)}{2}\left\langle g^{3}\right\rangle \varepsilon^{3}+\frac{\left(6 \Omega-\Omega^{3}\right)}{3 \sqrt{2 \pi}}\left\langle g^{4}\right\rangle \varepsilon^{4}+\ldots\right\}
$$

and:

$$
A_{2}(\Omega) \approx(1+\alpha)^{2} \rho \sqrt{\frac{k T}{m}}\left\{\sqrt{\frac{2}{\pi}}\left\langle g^{2}\right\rangle \varepsilon^{2}-\frac{3 \Omega}{2}\left\langle g^{3}\right\rangle \varepsilon^{3}+\frac{\left(3 \Omega^{2}-4\right)}{\sqrt{2 \pi}}\left\langle g^{4}\right\rangle \varepsilon^{4}+\ldots\right\}
$$

The steady state expressions for the moments are now readily obtained by solving the equations for the first two moments $\langle\Omega\rangle$ and $\left\langle\Omega^{2}\right\rangle$ at the steady state up to order $\varepsilon^{3}$. To lowest order in $\varepsilon$, we find $\langle\Omega\rangle=0$ and $\left\langle\Omega^{2}\right\rangle=(1+\alpha) / 2$. The next order of $\langle\Omega\rangle$ is obtained by replacing $\left\langle\Omega^{2}\right\rangle$ with $(1+\alpha) / 2$ in $\left\langle A_{1}(\Omega)\right\rangle$. Higher orders are obtained in this recursive manner, leading to the expressions (10) and (11). With this procedure, all the moments can be obtained to arbitrary order, although the algebra can become quite involved.

The Langevin equation, and its corresponding Fokker-Planck equation, can be obtained by breaking off the Kramers-Moyal expansion (A.2) after the first two terms, and by expanding the jump moments $A_{1}$ and $A_{2}$ up to order $\varepsilon^{3}$ (see the expressions above). In this way, we end up with the following linear Fokker-Planck equation:

$\partial_{t} P(\Omega, t)=-\partial_{\Omega}\left[\left(\frac{F}{\sqrt{I k T}}-\frac{\gamma}{I} \Omega\right) P(\Omega ; t)\right]+\frac{(1+\alpha)^{2}}{2} \rho \sqrt{\frac{2 k T}{\pi m}}\left\langle g^{2}\right\rangle \varepsilon^{2} \partial_{\Omega}^{2} P(\Omega ; t)$.

from which the resulting Langevin equation (19) can be derived.

\section{References}

[1] Goldhirsch I, 2003 Annu. Rev. Fluid Mech 35267

[2] Brilliantov N V and Pöschel T, 2004 Kinetic Theory of Granular Gases (Oxford: Oxford University Press)

[3] Zipelius A, 2004 Physica A 369143

[4] Jaeger H M, Nagel S R and Behringer R P, 1996 Rev. Mod. Phys. 681259

[5] Bug A L R and Berne B J, 1987 Phys. Rev. Lett. 75948

[6] Farkas Z, Tegzes P, Vukics and Vicsek T, 1999 Phys. Rev. E 607022

[7] Rapaport D C, 2001 Phys. Rev. E 64061304

[8] van der Meer D, Reimann P, van der Weele K and Lohse D., 2004 Phys. Rev. Lett. 92184301 
[9] van der Meer D and Reimann P, 2006 Europhys. Lett. 74384

[10] van Noije T P C and Ernst M H, 1998 Granular Matter 1, 57

[11] Brilliantov N V and Pöschel T, 2000 Phys. Rev. E 612809

[12] Martin P A and Piasecki J, 1999 Europhys. Lett. 46613

[13] Santos A and Dufty J W, 2006 Phys. Rev. Lett. 97058001

[14] Viot P and Talbot J, 2004 Phys. Rev. E 69051106

[15] Gomart H, Talbot J and Viot P, 2005 Phys. Rev. E 71051306

[16] Piasecki J and Viot P, 2006 Europhys. Lett. 741

[17] Piasecki J, Talbot J and Viot P, 2007 Phys. Rev. E 75051307

[18] Reimann P, 2002 Phys. Rep. 36157

[19] Cleuren B and Van den Broeck C, 2007 Europhys. Lett. 7750003

[20] Costantini G, Marini Bettolo Marconi U, and Puglisi A, 2007 Phys. Rev. E 75061124

[21] Servantie J and Gaspard P, 2007 Phys. Rev. Lett. 98266102

[22] Wang B Y and Král P, 2007 Phys. Rev. Lett. 98266102

[23] Van Kampen N G, 1992 Stochastic processes in physics and chemistry (Amsterdam: North Holland).

[24] Meurs P, Van den Broeck C and Garcia A, 2004 Phys. Rev. E 70051109

[25] Chapman S and Cowling T G, 1970 The mathematical theory of non-uniform gases (Cambridge: Cambridge University Press).

[26] Van den Broeck C, Kawai R and Meurs P, 2004 Phys. Rev. Lett. 93090601

[27] Van den Broeck C, Meurs P and Kawai R, 2005 New J. Phys. 710

[28] van den Broek M and Van den Broeck C, 2008 Phys. Rev. Lett. 100130601 